\title{
$\underline{\text { Reconfigurable optical fibre integrated phase change metadevices }}$
}

Yihao Cui ${ }^{1}$, Maximilian Maier ${ }^{2}$, Tiago Martins ${ }^{3}$, Davide Piccinotti ${ }^{4}$, Kevin F. MacDonald ${ }^{4}$, Nikolay I. Zheludev $^{4,5}$, and Behrad Gholipour ${ }^{1}$

1. Department of Electrical and Computer Engineering, University of Alberta, Canada

2. Ludwig-Maximilians-Univ. München (Germany)

3. INESC TEC and Department of Physics and Astronomy, Faculty of Sciences, University of Porto, Porto, Portugal

4. Optoelectronics Research Centre \& Centre for Photonic Metamaterials, University of Southampton, UK

5. Centre for Disruptive Photonic Technology, SPMS \& TPI, Nanyang Technological University, Singapore

Due to their small physical footprint, fibre integrated metamaterials and metadevices made from phase change chalcogenide semiconductors that can be dynamically reconfigured using optical or electrical stimuli present the most promising platform for integration into future telecommunication networks to alleviate the data latency and high power consumption associated with current network configurations. Here, through numerical simulations, we present reconfigurable metadevices that can be integrated onto the tip and side of commercial optical fibres showing tunable behavior across the entire telecommunication band. Such devices can be used for dynamic dispersion control and signal switching. 